\title{
\begin{tabular}{l|l} 
POLITIQUES \& & Politiques et management public
\end{tabular}

\section{Le sport dans la stratégie de communication des collectivités territoriales : le cas de la Seine-Saint- Denis}

Sport in territoritorial communication strategy: the Seine-Saint-Denis's case

\section{Christopher Hautbois et Michel Desbordes}

\author{
CpenEdition \\ Journals \\ Édition électronique \\ URL : http://journals.openedition.org/pmp/4480 \\ ISSN : 2119-4831 \\ Éditeur \\ Institut de Management Public (IDPM) \\ Édition imprimée \\ Date de publication : 15 décembre 2011 \\ Pagination : $509-530$ \\ ISSN : 0758-1726 \\ Référence électronique \\ Christopher Hautbois et Michel Desbordes, «Le sport dans la stratégie de communication des \\ collectivités territoriales : le cas de la Seine-Saint-Denis », Politiques et management public [En ligne], Vol \\ 28/4 | 2011, mis en ligne le 13 avril 2014, consulté le 30 avril 2019. URL : http:// \\ journals.openedition.org/pmp/4480
}




\title{
Le sport dans la stratégie de communication des collectivités territoriales : le cas de la Seine-Saint-Denis
}

\author{
Christopher Hautbois ${ }^{a^{*}}$ et Michel Desbordes ${ }^{b}$ \\ a Maître de conférences, ISC Paris, school of management, \\ 14, bd du Fort-de-Vaux, 75017 Paris \\ ${ }^{b}$ Professeur des Universités, ISC Paris, school of management, \\ 14, bd du Fort-de-Vaux, 75017 Paris
}

\section{Résumé}

Depuis les années 1990, le marketing territorial apparait comme un domaine de recherche original, fécond bien que peu exploré. Les travaux de Sperling (1991; 1995), Girard (1999) mais aussi de Cohen-Bacrie (2003) et Hatem (2007) ont progressivement structuré le cadre de cette réflexion. Le marketing territorial consiste, pour reprendre la définition de Hatem (2007), à « améliorer la part de marché d'un territoire donné dans les flux nationaux et internationaux de différentes natures ». Pour atteindre un tel objectif, les élus locaux doivent concevoir une stratégie de communication adaptée. Différents leviers peuvent dès lors être mobilisés par les manageurs publics. Le sport en général apparaît à leurs yeux comme un vecteur pertinent de communication territoriale.

Cet article vise à interroger le rôle stratégique des acteurs publics dans la construction d'une communication territoriale. II s'appuie sur une étude longitudinale (10 ans) portant sur le département de Seine-Saint-Denis. Des entretiens semi-directifs ont été réalisés auprès des responsables publics ayant été au cœur de la politique de communication durant la décennie 1998-2008. Leur analyse fait apparaître quatre rôles principaux : I'acteur public « spectateur », l'acteur public « opportuniste », I'acteur public « accompagnateur » et l'acteur public «moteur».

Mots clés : management public, communication, sport, image, stratégie, collectivités locales, Seine-Saint-Denis

\footnotetext{
*Auteur correspondant : chautbois@iscparis.com doi : 10.3166/pmp.28.509-530 @ 2011 IDMP/Lavoisier SAS. Tous droits réservés
} 


\section{Abstract}

Sport in territoritorial communication statégy: the Seine-Saint-Denis's case. From the 1990's, urban marketing became in France an original field of research but not much explored. Researches conducted by Sperling (1991, 1995), Girard (1999) but also Cohen-Bacrie (2003) progressively designed a theoretical framework for this field. According to Hatem (2007), the territorial marketing consists in "improving the market share in national or international business for a given territory". To reach such a goal, public officials have to design a relevant communication strategy for their own territory. Different opportunities may exist included sporting events.

This article aims to study the strategic action of public officials in this territorial communication. A long-term study has been driven (10 years) on the Seine-Saint-Denis department's case. Interviews with all the public officials of the department involved in this strategy have been conducted. Data analysed suggest four different strategic options for public officials: public official as a "spectator" of the communication strategy, as an "opportunist", as a "facilitator" or as a "leader".

(c) 2011 IDMP/Lavoisier SAS. Tous droits réservés

Keywords: public management, communication strategy, sport, image, public official, Seine-Saint-Denis

\section{Introduction}

Les mutations vécues par les collectivités locales françaises depuis les années 1980 ont repensé la manière de gouverner les territoires. Dans un contexte de concurrence interterritoriale accrue, les élus locaux apparaissent de plus en plus comme des manageurs publics devant fixer des objectifs de développement de leur territoire dans un calendrier établi. Ce contexte concurrentiel les oblige à développer un marketing territorial afin « d'améliorer la part de marché d'un territoire donné dans les flux nationaux et internationaux de différentes natures (commerce, investissement, tourisme, compétences) » (Hatem, 2007). La stratégie alors développée pour atteindre ces objectifs doit tenir compte de différents paramètres (économiques, sociaux, environnementaux, fiscaux, légaux, etc.) endogènes ou exogènes au territoire. L'amélioration de cette «part de marché du territoire » passe également par la définition d'une communication territoriale spécifique (Houllier-Guibert, 2009). Celle-ci repose sur l'identification d'objectifs précis, de public-cible et s'appuie sur la formulation d'un message et sur le choix de sa tonalité. Dans ce contexte, le sport en général et les événements sportifs en particulier apparaissent comme un vecteur pertinent de cette communication des territoires.

L'objectif n'est pas ici d'analyser ce qui fonde une bonne stratégie de communication des territoires (bonnes pratiques), ni d'évaluer l'efficacité d'une stratégie même si ces deux points peuvent faire l'objet de réflexions utiles aux acteurs. Dans le cadre d'une démarche exploratoire, il s'agit ici d'étudier le rôle stratégique des acteurs publics dans la construction d'une communication territoriale par le sport. Cet article étudie, sur une période longue (1998-2008), le cas particulier du conseil général de Seine-Saint-Denis. Situé au nord de Paris, ce département urbain souffre depuis de nombreuses années d'une mauvaise réputation avec en point d'orgue les émeutes urbaines de novembre 2005. Des entretiens ont été menés 
et retranscrits, avec l'ensemble des acteurs publics locaux impliqués dans la communication territoriale par le sport durant cette période. L'analyse de contenu réalisée fait apparaître que selon la période, le contexte local et l'événement sportif organisé sur ce territoire, les élus de Seine-Saint-Denis peuvent adopter quatre rôles différents dans la communication territoriale : «spectateur», « opportuniste », « accompagnateur» et « moteur».

Après avoir montré le rôle de la communication dans les stratégies des collectivités territoriales, l'article analysera les discours de leurs concepteurs, pour ensuite préciser les stratégies de communication menées au travers de quelques événements sportifs notables. Une typologie des rôles stratégiques de l'acteur public tentera de synthétiser les investigations menées.

\section{La communication des collectivités territoriales : des stratégies en cours de développement}

Cette première partie rappelle en quoi le contexte dans lequel évoluent les collectivités territoriales a profondément changé depuis trente ans et qu'il constitue le principal facteur ayant favorisé l'émergence d'un marketing des territoires. Cette partie souligne également l'émergence progressive du sport comme vecteur de communication.

\subsection{Mutations de l'organisation territoriale française et stratégies de marketing territorial}

Les lois de décentralisation ont augmenté le degré de liberté dont jouissent les élus locaux dans la manière de développer et d'animer leur territoire. Cette autonomie se décline au travers de politiques économiques, sociale, culturelle, sportive, etc. propres à chaque espace. Cette liberté a mécaniquement accru la concurrence entre les territoires qui doivent dès lors trouver les leviers permettant de tirer leur épingle du jeu. Ceci explique également la volonté des acteurs locaux de créer les conditions favorisant le développement territorial (Lacour, 2005) sur la base de caractéristiques territoriales perçues comme spécifiques (Peirce, Brent, 2007). Bouinot (2004) va dans ce sens en évoquant, pour les collectivités, la recherche d'un « futur voulu plutôt [qu'un] futur subi ». La nécessité de développer de véritables stratégies de marketing public se fait jour depuis les années 1990 (Bloom, 1997; Burton, 1999; Buurman, 2001). Ce champ de réflexion a fait l'objet de travaux importants depuis cette période : Texier, Valla (1992), Sperling (1995), Noisette, Vallerugo (1996), Girard, (1999), Godron (2003) notamment.

Hatem (2007) définit pour sa part les principes et méthodes du marketing territorial. Il accorde une place centrale à l'objectif de développement économique du territoire et voit dans le marketing l'outil permettant de l'atteindre. En tant que point d'appui classique du marketing, la communication occupe une place stratégique en définissant traditionnellement plusieurs objectifs : développement économique du territoire, notoriété, image et/ou insertion sociale. La définition du public cible constitue également une phase importante. Ces plans de communication peuvent viser la population ${ }^{1}$ et les entreprises locales (communication

\footnotetext{
${ }^{1}$ Qui peut être amené à quitterun territoire, en cas de désaccord avec la politique générale de développement qui y est menée, selon le principe de « vote par les pieds » proposé par Tiebout (1956).
} 
interne) mais aussi les touristes, la population et entreprises extérieures au territoire (communication externe). Dans l'ensemble, il s'agit d'attirer et de maintenir sur le territoire les agents économiques à la base du développement territorial (Texier, 1999) même si pour Cohen-Bacrie (2003), « le marketing territorial s'inscrit dans une problématique plus large qui le mêle aux notions de développement local et de stratégie d'image ».

Un dernier aspect de la communication territoriale tient à la déclinaison du message et au choix de supports les plus appropriés : affichage, spots TV ou radio, internet, Web 2.0, etc. Dans cet article, il ne s'agit pas de dire lesquels sont les meilleurs ou les plus efficaces ${ }^{2}$. Le tableau ci-dessous regroupe toutefois des exemples de campagnes récemment développées par des collectivités et qui en constituent des illustrations pertinentes.

Tableau 1 : Liste non-exhaustive de campagnes de communication développées par des collectivités territoriales

\begin{tabular}{|c|c|c|c|}
\hline $\begin{array}{l}\text { Nom de la } \\
\text { collectivité }\end{array}$ & Type & $\begin{array}{l}\text { Nom } \\
\text { de la compagne }\end{array}$ & Date \\
\hline Le Havre & Ville & Je vis ailleurs (jevisailleurs.com) & $2008-2010$ \\
\hline Metz & Ville & Je veux Metz (jeveuxmetz.com) & 2010 \\
\hline Reims & Ville & $\begin{array}{l}\text { Invest in Reims (qui est aussi le nom de l'agence } \\
\text { chargée du développement économique de la } \\
\text { ville) (investinreims.com) }\end{array}$ & 2011 \\
\hline Orne & Département & $\begin{array}{l}\text { Je m'installe dans l'Orne en Normandie } \\
\text { (jeminstalledanslorneen normandie.com) }\end{array}$ & 2010 \\
\hline Bretagne & Région & $\begin{array}{l}\text { Tourisme Bretagne } \\
\text { (tourismebretagne.com) }\end{array}$ & 2010 \\
\hline
\end{tabular}

Notons enfin que le contexte de ces communications territoriales varie. Elles peuvent correspondre à une volonté des élus de planifier, à moyen ou long terme, la construction d'une image du territoire jugée positive ou valorisante (approche pro-active). Ces campagnes peuvent également intervenir en réaction à un événement de nature à porter préjudice à l'image d'un territoire et, par ricochet, à son développement économique (approche défensive). Ce fut par exemple le cas pour le département de la Mayenne quand, en 2001, l'épidémie de fièvre aphteuse, largement couverte par les médias, avait entraîné la destruction de nombreux troupeaux de bétails) ou, pour la région Aquitaine, la marrée noire consécutive au naufrage de l'Erika en 2004 avait fait porté un risque sur l'image de ce territoire et sur le bon déroulement de la saison touristique)

\footnotetext{
${ }^{2}$ Bien que cela constitue un enjeu important et que la phase d'évaluation représente une étape à part entière de la communication territoriale.
} 


\subsection{Le sport comme levier de communication territoriale}

Aujourd'hui, les acteurs publics locaux cherchent de plus en plus à construire une identité propre à leur territoire (Rebillard, 2007). Certains mettent ainsi en avant un atout ou un savoirfaire spécifique : l'industrie de la plaisance à La Rochelle, la recherche technologique à Grenoble, l'image et le multimédia en Ile-de-France, le nucléaire en Bourgogne, la céramique dans le Limousin ${ }^{3} \ldots$ Les secteurs potentiels sur lesquels fonder cette stratégie de communication sont donc très vastes. Le sport au sens large constitue aujourd'hui l'un d'entre eux. Bien que les lois de décentralisation des années 1980-1990 aient été muettes dans ce domaine, l'investissement des collectivités territoriales n'est pas nouveau. Chazaud (1989) l'analysait déjà il y a 20 ans. Aujourd'hui, il constitue un élément structurant du système sportif français et était estimé en 2007

à 16,4 milliards d'euros ${ }^{4}$, soit environ la moitié du poids total de l'économie du sport en France (33 milliards).

Néanmoins, jusqu'à maintenant, le sport amateur ou professionnel a essentiellement été considéré comme un facteur d'animation du territoire ${ }^{5}$. Son utilisation n'a que très récemment été envisagée dans le cadre d'une véritable stratégie de communication du territoire (Chappelet, 2006; Ferrand, Chanavat, 2006). Ici, les événements sportifs de dimension nationale voire internationale apparaissent comme une vitrine pertinente pour les collectivités ${ }^{6}$. Outre ceux directement organisés à l'initiative des acteurs publics locaux ou ceux dont ils sont les partenaires, les collectivités territoriales peuvent se por-

ter candidates à l'organisation d'un événement sportif dont les droits sont détenus par une institution : Fédérations sportives nationales, Comité International Olympique (CIO) pour les Jeux Olympiques (JO), International Association of Athletics Federations (IAAF) pour les Championnats du monde d'athlétisme, Larivière organisation pour le Tour de France à la voile... Au demeurant, les travaux sur ce thème sont à la fois récents et rares. Le tableau 2 en fournit une liste non-exhaustive.

\footnotetext{
${ }^{3}$ Certains de ces savoir-faire territoriaux ont d'ailleurs été organisés autour de pôles de compétitivité. Voir le site www.competitivite.gouv.fr.

${ }^{4}$ Stat-Info n09-02 de septembre 2009.

${ }^{5}$ Voir Bayeux $(2006 ; 2009)$ pour un panorama des relations entre les collectivités territoriales et le sport. ${ }^{6}$ À titre d'exemple, alors qu'ASO, organisateur du Tour de France, sélectionne chaque année une trentaine de villes devant accueillir une étape du Tour, ce sont près de 300 collectivités qui candidatent. Une attente de 3 à 5 ans avant d'accueillir le passage du Tour constitue par ailleurs la règle.
} 
Tableau 2 : Liste non-exhaustive des travaux analysant les stratégies de promotion menées par des collectivités locales utilisant les événements sportifs

\begin{tabular}{|c|c|c|c|c|}
\hline Localisation & $\begin{array}{l}\text { Type de } \\
\text { structure } \\
\text { publique }\end{array}$ & $\begin{array}{l}\text { Événement ou } \\
\text { spectacle sportif } \\
\text { utilisé }\end{array}$ & $\begin{array}{l}\text { Objectif } \\
\text { stratégique }\end{array}$ & Références \\
\hline $\begin{array}{l}\text { Montréal } \\
\text { (Canada) }\end{array}$ & Ville & $\begin{array}{l}\text { Ville hôte des } \\
\text { Canadiens de } \\
\text { Montréal, équipe } \\
\text { professionnelle } \\
\text { de hockey } \\
\text { sur glace }\end{array}$ & $\begin{array}{l}\text { Associer l'image } \\
\text { de la ville à celle } \\
\text { prestigieuse de } \\
\text { cette équipe } \\
\text { sportive } \\
\text { professionnelle }\end{array}$ & Richelieu (2008) \\
\hline $\begin{array}{l}\text { Rennes } \\
\text { (France) }\end{array}$ & Ville & $\begin{array}{l}\text { «La nuit du sport », } \\
\text { événement organise } \\
\text { par la ville }\end{array}$ & $\begin{array}{l}\text { Renforcer le lien } \\
\text { social grâce à un } \\
\text { événement sportif } \\
\text { original }\end{array}$ & Lebreton (2008) \\
\hline Turin (Italie) & Ville & $\begin{array}{l}\text { Ville hôte des JO } \\
\text { d'hiver } 2006\end{array}$ & $\begin{array}{l}\text { Utiliser les JO } \\
\text { pour améliorer } \\
\text { les infrastructures } \\
\text { du territoire } \\
\text { et développer } \\
\text { l'activité } \\
\text { touristique }\end{array}$ & $\begin{array}{l}\text { Mela \& al (2005), } \\
\text { Burgay (2005) }\end{array}$ \\
\hline $\begin{array}{l}\text { Vendée } \\
\text { (France) }\end{array}$ & Département & $\begin{array}{l}\text { Vendée Globe } \\
\text { Partenaire de } \\
\text { l'équipe cycliste } \\
\text { professionnelle } \\
\text { Bouygues Telecom }\end{array}$ & $\begin{array}{l}\text { Promouvoir } \\
\text { le territoire } \\
\text { pour développer } \\
\text { l'activité } \\
\text { touristique }\end{array}$ & $\begin{array}{l}\text { Hautbois \& } \\
\text { Desbordes (2008) }\end{array}$ \\
\hline $\begin{array}{l}\text { Valais } \\
\text { (Suisse) }\end{array}$ & Canton & $\begin{array}{l}\text { Ville candidate à } \\
\text { l'accueil des Jeux } \\
\text { Olympiques d'hiver } \\
\text { de } 2006 \\
\text { Ville d'accueil du } \\
\text { Masters européen de } \\
\text { Golf, du Grand Raid } \\
\text { Cristalp, du Tour de } \\
\text { Romandie, du Tour } \\
\text { de Suisse }\end{array}$ & $\begin{array}{l}\text { Promouvoir le } \\
\text { territoire pour } \\
\text { développer } \\
\text { l'activité } \\
\text { touristique }\end{array}$ & Seppey (2005) \\
\hline $\begin{array}{l}\text { Rhône-Alpes } \\
\text { (France) }\end{array}$ & Région & $\begin{array}{l}\text { Région hôte de trios } \\
\text { Jeux Olympiques } \\
\text { d'hiver (1924, } \\
\text { 1968, 1992), de } \\
\text { l'épreuve cycliste du } \\
\text { Dauphiné-Libéré }\end{array}$ & $\begin{array}{l}\text { Promouvoir } \\
\text { le territoire } \\
\text { pour développer } \\
\text { l'activité } \\
\text { touristique }\end{array}$ & Garde (2005) \\
\hline
\end{tabular}

Source-Les auteurs

Compte tenu de la place centrale des acteurs publics dans le système local que constitue une collectivité, l'analyse de leur rôle stratégique dans le cadre d'une communication par le sport présente un intérêt important. 


\section{L'analyse du discours des acteurs publics, concepteurs de la communication territoriale}

La méthode employée pour cette recherche est à la fois qualitative (entretiens) et documentaire. Elle est présentée de manière détaillée en annexe B. Cette partie rappelle que l'objectif de recherche est d'analyser le rôle des acteurs publics locaux dans la construction de la communication d'un territoire spécifique, celui de Seine-Saint-Denis.

\subsection{Objectif : analyse du rôle stratégique des acteurs publics locaux}

Le sport apparaît donc comme un support pertinent de la communication des territoires. Comme cela a été évoqué précédemment, l'objectif n'est pas ici d'analyser ce qui fonde une bonne stratégie de communication par le sport des territoires selon le principe de best practices. Il ne s'agit pas non plus d'identifier les déterminants d'une stratégie efficace qui nécessiterait de comparer la notoriété ou l'image d'un territoire avant et après la mise en œuvre d'un plan de communication. Il s'agit plus spécifiquement d'analyser la construction d'une politique de communication territoriale par le sport sur une période longue.

Notre démarche repose sur l'analyse détaillée d'un seul cas, au sens d'Eisenhardt (1989) ou de Yin (2003), celui du département de la Seine-Saint-Denis et porte sur une période longue (1998-2008) ${ }^{7}$. Elle présente un caractère exploratoire. De ce fait, il n'y a pas de volonté de représentativité statistique du cas étudié : la Seine-Saint-Denis présente des singularités qui font que les résultats obtenus ne sont pas immédiatement transposables à d'autres collectivités. Cette démarche semble néanmoins pertinente afin de faire émerger, par le biais d'entretiens approfondis, les comportements stratégiques des élus locaux. La répétition de ces observations conduira à une modélisation progressive de ces résultats. En conséquence, cette recherche s'inscrit essentiellement dans une logique empirico-inductive.

\subsection{La Seine-Saint-Denis, un département urbain à la mauvaise réputation}

Le dynamisme industriel de ce département a toujours donné lieu à d'importantes vagues d'immigration venues d'abord du sud et de l'ouest de la France, puis d'Espagne, du Portugal et enfin d'Afrique subsaharienne. Conséquence de cette histoire singulière, la Seine-Saint-Denis est aujourd'hui le département français qui compte le plus de population immigrée. Il s'agit là d'un aspect important du département avec lequel doivent composer les élus locaux dans leur stratégie de développement. Outre cet aspect lié à la structure de sa population, la Seine-Saint-Denis se caractérise aujourd'hui par une part de main-d'œuvre non-qualifiée, de population non-diplômée et un taux de chômage supérieurs à la moyenne nationale. Une partie de la population de ce département se trouve ainsi en difficulté sociale et fait de la Seine-Saint-Denis un territoire « sensible».

\footnotetext{
${ }^{7}$ L'année 1998 coïncide avec l'inauguration du Stade de France et l'organisation en France de la Coupe du monde de football. 2008 correspond à la phase de bilan de la Coupe du Monde de rugby achevée en octobre 2007. 1998-2008 permet l'analyse d'une décennie de communication publique par le sport qui s'achève avec les élections cantonales de 2008.
} 


\section{Encadré 1 - Fiche signalétique du département de Seine-Saint-Denis}

Région : Ile-de-France

Préfecture : Bobigny

Population totale : 1,5 million (1999)

Superficie : $236 \mathrm{~km}^{2}$

Densité de population : 6182 habitants $/ \mathrm{km}^{2}$. La Seine-Saint-Denis est le $2^{\mathrm{e}}$ département de France derrière les Hauts-de-Seine du point de vue de la densité de population

Outre ces déterminants structurels, le département de Seine-Saint-Denis a vécu à l'automne 2005 une importante période d'émeutes urbaines. Quelle que soit l'origine de ces tensions, le fait qu'elles soient relayées par les médias du monde entier a contribué à renforcer la mauvaise réputation dont souffre parfois ce département du fait des tensions et des conflits qu'il connaît et de l'économie parallèle qui s'y développe.

\section{Une stratégie de communication du territoire basée sur les événements sportifs}

Cette partie rend compte de la manière dont les élus de Seine-Saint-Denis ont utilisé, au cours de la décennie étudiée (1998-2008), le sport dans le cadre de leur plan de communication. Les données secondaires collectées ainsi que les entretiens réalisés indiquent que ce département a essentiellement fondé sa stratégie sur les événements sportifs ${ }^{8}$ : la Coupe du monde de football (1998), Championnats du monde (2003), Coupe du monde de rugby (2007), Transat AG2R (2006).

\subsection{Coupe du monde 1998 de la FIFA, l'opportunité pour les acteurs publics locaux de démontrer un savoir-faire territorial}

La France n'avait pas organisé la coupe du monde de football depuis 1938. Sa capacité à assurer le bon déroulement de la compétition est essentielle tant pour le comité d'organisation que pour la France alors engagée alors dans la course à la candidature pour l'accueil des Jeux Olympiques d'été de 2008.

"C'est vrai que c'était un gros challenge pour nous de participer à un tel événement.

Tout ce qui était organisé sur notre territoire donnait lieu à une exposition médiatique très importante » [PMS1]

L'organisation de cette épreuve était également un défi important pour les architectes du Stade de France, construit pour l'occasion, et qui était pour la première fois soumis à des contraintes répétées durant quatre semaines ( 9 matchs dont un quart-de-finale, une demi-finale et la finale) et pour le monde sportif dans son ensemble.

"Nous n'avions pas organisé en France un tel événement et des matches aussi importants depuis très longtemps. Il était très important que les matches organisés dans le nouveau stade soient un succès » [PMS1]

La démonstration de leur savoir-faire est également importante pour les élus de Seine-Saint-

\footnotetext{
${ }^{8}$ Voir également l'annexe $C$.
} 
Denis. Ces derniers reçoivent en effet de l'État, engagé dans l'événement, une double mission : la gestion du public et la lutte contre un éventuel sentiment d'exclusion de la population. Le premier volet consiste en un accompagnement social de tous les publics. L'objectif non dissimulé est que la population locale ne contrecarre pas le bon déroulement de la compétition. L'ensemble du mouvement sportif du département est ainsi mobilisé. Le second objectif est celui consistant à permettre à la population du département de s'approprier l'événement :

"Il s'agit de lutter contre toute situation de proximité géographique avec l'événement, c'est-à-dire le fait d'habiter à côté du Stade de France, mais d'exclusion économique et sociale, c'est-à-dire de ne pas avoir les moyens d'assister aux matches, qui peut concerner une partie de la population locale » [LSD]

En contrepartie de cette mission, l'État, via le comité d'organisation de la Coupe du monde, a donné au département un accès prioritaire à la billetterie afin que les acteurs publics locaux puissent organiser des opérations de relations publiques durant les matches avec des partenaires économiques ou institutionnels clés. Après l'échec de la candidature de Paris pour les Jeux Olympiques d'été en 2008, la France s'est de nouveau portée candidate à l'organisation des JO de 2012. Cette candidature s'est notamment appuyée sur la réussite de l'organisation de la Coupe du monde 1998 qui était un élément stratégique pour les porteurs politiques du projet, notamment ceux du département de Seine-Saint-Denis, de la région Ile-de-France et de Paris.

«Durant la Coupe du monde 1998, nous savions que le monde entier nous regardait, notamment les membres votants du Comité International Olympique qui étaient supposés désigner la ville hôte des Jeux Olympiques de 2012 quelques années plus tard » [SLD]

\subsection{Championnats du monde d'athlétisme 2003, une étape du processus de reconquête en Seine-Saint-Denis}

Les Championnats du monde d'athlétisme sont organisés tous les deux ans. Ceux de 2003 l'ont été intégralement au Stade de France. Pour cet événement, dont la participation financière de la sphère publique a été particulièrement importante (50\% du budget total), les élus de Seine-Saint-Denis ont reçu du comité d'organisation la mission de contribuer au remplissage du stade. Remplir durant 10 jours un stade de 60000 personnes $^{9}$ (soit 600 $000 \mathrm{au}$ total) venant assister au « seulement» dixième sport le plus pratiqué en France au nombre de licenciés constitue un défi important.

"Nous avons été très impliqués dans cet événement d'un point de vue financier, sur le plan de l'organisation, notamment en termes de logistique, mais aussi par rapport au business de l'événement » [PMS2]

Là encore, la contrepartie proposée par le comité d'organisation a été l'accès du conseil général à la billetterie. Celui-ci a notamment pu organiser des opérations à destination des établissements scolaires du département. Le Comité d'expansion économique du 93 (Comex 93) a quant à lui réalisé des opérations de relations publiques en invitant des prospects à assister à plusieurs épreuves de ces Championnats du monde

"Grâce à notre implication, nous avons organisé plusieurs opérations en parallèle de l'événement, ce qui était vraiment très bien, même si les championnats du monde d'athlétisme ne sont pas aussi importants qu'une coupe du monde de football » [SLD]

\footnotetext{
${ }^{9} 81000$ en configuration football/rugby. 60000 en configuration athlétisme.
} 


\subsection{Coupe du monde de rugby 2007 , la Seine-Saint-Denis carrefour du monde sportif}

S'agissant de la Coupe du monde de rugby 2007, organisée en France du 7 septembre au 20 octobre, la problématique est un peu différente. D'une part, le prix élevé des places permettait difficilement à une structure publique d'acheter au prix de vente officiel des packages ou des lots pour inviter des personnalités, des écoles ou des clients. En outre, l'organisation de la Coupe du monde prévoit la mise à disposition gratuite, par les villes, de leur stade au Groupement d'intérêt Public (GIP)-organisateur. Les collectivités territoriales se sont donc retrouvées « dépossédées de leur équipement » le temps de la compétition et sont devenues clientes de leur propre stade. Par ailleurs, le département de Seine-Saint-Denis n'a pas été sollicité par l'organisation dans le cadre d'une mission d'accompagnement social du public (comme pour la Coupe du monde de football), ni pour favoriser le remplissage du Stade de France (comme pour les Championnats du monde d'athlétisme), le GIP estimant ne pas avoir besoin des élus locaux pour ces deux objectifs. Par conséquent, comment le département d'accueil peut-il faire pour que la Coupe du monde ne soit pas perçue par la population locale comme un événement uniquement visible à la TV ? Comment être présent sur l'événement sans disposer de son stade et sans être directement impliqué dans son organisation? Les élus de Seine-Saint-Denis, en partenariat avec diverses villes du département, ont fait une proposition afin d'être présent en parallèle dans la compétition.

"Le modèle économique de la Coupe du monde de rugby est très particulier et c'est très difficile pour nous d'être au cœur de l'événement. En tant que collectivité locale, nous étions supposés être partie prenante de l'événement mais dans les faits, nous ne pouvions qu'organiser des animations en parallèle de la manifestation en tant que telle. C'est finalement que nous avons fait » [PMS3]

En effet, le département de Seine-Saint-Denis, et plus particulièrement la ville de Noisyle-Grand, ont accueilli le « camp de base » de l'équipe d'Afrique du Sud durant la compétition (le coût moyen pour accueillir une équipe en résidence est d'environ 300 000,00 euros). Ceci lui donne le statut de département hôte de la Coupe du Monde de Rugby 2007 (le label qui lui est associé est utilisé dans toute la communication du conseil général liée à l'événement) et lui donne accès à la billetterie. Les 7800 places achetées permettront aux jeunes et aux clubs de rugby investis dans les projets d'accompagnement d'assister à l'un des 7 matchs (sur les 48 que compte la compétition) se déroulant au Stade de France. Le conseil général a complété son offre d'accueil par un programme « Host Family » qui s'adresse aux femmes et enfants des joueurs. Ce programme comprend l'hébergement des familles, des visites de Paris et du patrimoine culturel francilien, la découverte de la gastronomie française, des cours de français, des activités de sport et de loisirs.

Outre l'aspect sportif (l'équipe fait partie des meilleures mondiales), le choix d'accueillir l'Afrique du Sud s'explique pour plusieurs raisons : historique (durant l'apartheid, de nombreux afrikaners ont été hébergés dans le département), symbolique (à des échelles différentes, la Seine-Saint-Denis et l'Afrique-du-sud sont des terres de métissage culturel mais aussi de lutte contre la discrimination), politique (des liens existent entre les représentants du département et de l'Afrique du Sud) et médiatique (la présence des médias assistant aux matches d'entraînement de l'équipe est l'occasion de montrer que la Seine-Saint-Denis est un territoire comme les autres :

"Nous aspirons à la normalité » [PMS3] 


\subsection{La Transat AG2R ou comment associer un département urbain à une compétition de voile}

La Transat AG2R est une course au large longue d'environ trois semaines permettant de rallier Concarneau à Saint-Barthélemy dans les Antilles françaises. La Transat AG2R est la seule course transatlantique en double qui présente la caractéristique de mettre tous les concurrents sur un pied d'égalité puisque tous courent sur des bateaux identiques (Figaro Bénéteau II). La 8 e édition est partie le 9 avril 2006 avec 28 équipages au départ.

Cette course à la voile occupe un statut particulier dans le plan de communication du département dans la mesure où celui-ci ne constitue pas le territoire d'accueil de la compétition et qu'il n'en est pas non plus l'organisateur. Il en est l'un des participants. L'idée de participer à cette transat est née en automne 2005, durant la période de violence urbaine à laquelle a été confronté le département. Les élus locaux ont souhaité lutter contre l'image négative du département qui en a découlé. Il a donc été décidé d'engager le bateau «Esprit 93 », aux couleurs du département, dans la compétition. Ce projet est justifié par le fait qu'il possède les valeurs que les élus locaux veulent voir associer au département : «ambition, solidarité, courage, innovation, solidarité et fraternité » (source : dossier de partenariat)

« Notre idée était de mettre la Seine-Saint-Denis là où on ne l'attendait pas » [ESEP2]

D'après les manageurs publics, trois facteurs principaux ont permis d'intégrer cet événement au plan de communication du département :

- le sport correspond à des valeurs de jeunesse : l'association entre sport et jeunesse a été considérée comme un postulat de base dans la stratégie. Or la jeunesse est l'une des caractéristiques importantes du département de Seine-Saint-Denis. Dans le projet de participation à la Transat, cette jeunesse a été incarnée par la skippeur du bateau, Marine Chombart de Lauwe, alors âgée de 25 ans.

- la voile pour ses valeurs : les acteurs locaux ont jugé que la Seine-Saint-Denis et la Transat AG2R avaient plusieurs valeurs en partage.

"Ces valeurs sont à la fois celles du département mais aussi celles qu'il faut pour s'engager dans ce type de course à la voile : la solidarité est nécessaire en mer, mener à bien ce projet nécessite d'aller de l'avant, d'être entreprenant et la transat reste une compétition sportive. Il faut avoir des objectifs ambitieux. Le nôtre était de terminer dans les 10.» [ESEP2]

- les répercussions médiatiques de l'événement : en moyenne, à chaque édition correspond 12 heures d'antenne TVA, dont une partie retransmise sur France 3, France 2, TF1, 8 heures d'antenne radio dont France Bleu, Sport FM, France Info représentant plus de 300 passages et près de 900 articles de presse de mars à juin

Construit autour des mots-clés de jeunesse, de dynamisme et visant notamment un objectif d'animation et de valorisation de l'image du territoire, le projet «Esprit 93 » a donné lieu à des opérations menées en accord avec l'association Promovoile 93. Les élèves de 33 collèges du département ont ainsi participé à des opérations d'initiation à la voile et à des rencontres éducatives avec les skippeurs du bateau. La 14 édition du Trophée des Bahuts a été organisée en juin 2006. Ce trophée consiste en un rallye de deux jours sur des bateaux de 10 à 12 mètres ayant réuni 14 établissements scolaires et 168 jeunes du département. La participation du monde éducatif de Seine-Saint-Denis à ce projet a donc été l'un des axes d'exploitation de l'événement. 
Parallèlement, la volonté de promouvoir le département a donné lieu à des opérations de communication visant deux types de publics : les acteurs économiques et les institutionnels d'une part, le grand public, les habitants du département d'autre part. Ainsi, lors du Marché international des professionnels de l'immobilier (MIPIM) organisé en mars 2006 au Palais des festivals de Cannes, les acteurs du département ont valorisé le projet sur leur stand et organisé des rencontres entre les skippers et d'éventuels partenaires. Pour la promotion grand public, le PC course a été installé dans les locaux du département à Bobigny avec la présence d'un animateur-navigateur, de panneaux d'affichages dédiés, d'espaces de communication et la projection de film sur grand écran. Ce PC était ouvert au public afin qu'il puisse suivre la course et interagir avec les skippeurs. 80 personnes ont ainsi assisté au départ de la course et 100 à la cérémonie de clôture de l'événement le 27 avril 2007.

Enfin, pour compléter ses actions de communication grand public, plusieurs supports ont été mobilisés : plaquettes, affiches, autocollants, cartes postales, goodies, panneaux, autoportants au PC course, reportage photo et vidéo, rubrique internet dédiée, réalisation d'une maquette du bateau à l'échelle $1 / 20^{\mathrm{e}} \ldots$

\section{Quatre rôles stratégiques de l'acteur public}

Cette recherche exploratoire portant sur le cas de la Seine-Saint-Denis est basée sur la réalisation exhaustive d'entretiens avec les décideurs publics locaux ayant piloté la communication du département au cours de la décennie 1998-2008. Ces entretiens ont notamment permis d'étudier le rôle stratégique de ces acteurs publics. L'analyse des données recueillies et en particulier celles relatives aux actions menées par les élus locaux durant dix ans tend à faire émerger quatre rôles stratégiques en matière de communication territoriale par le sport.

\subsection{L'acteur public « spectateur »}

Le Stade de France constitue, de part sa taille (81 000 places), de part le symbole qu'il représente pour les Français depuis la victoire de l'équipe de France lors de la Coupe du Monde de football 1998, de part son rayonnement international et le nombre de manifestations sportives ou culturelles organisées chaque année (30 environ), un équipement unique en France. Pour les acteurs publics de Seine-Saint-Denis, posséder un tel équipement sur leur territoire apparaît a priori comme une opportunité unique de communication en leur donnant l'occasion de s'associer à des événements prestigieux. La présence durable de ce stade sur leur territoire s'assimilerait à une « rente de situation » leur fournissant la certitude de pouvoir être le territoire d'accueil de ces manifestations. Deux remarques doivent ici être faites. D'une part, les entretiens réalisés ont montré qu'au début des années 1990, les acteurs publics locaux ont mis tout leur poids politique pour obtenir de l'État que ce futur grand stade inauguré en 1998 voit le jour en Seine-Saint-Denis. La « rente de situation » supposée dont jouiraient les acteurs publics locaux ne serait donc pas le fruit d'un heureux hasard mais au contraire le résultat d'un fort lobbying initié dès le début du projet et ayant permis la construction de ce Stade dans le département et non à Sénart comme cela était envisagé à l'origine. 
D'autre part, le Stade de France, et donc indirectement la Seine-Saint-Denis en tant que territoire d'implantation, accueille chaque année plusieurs événements qui constituent a priori autant d'opportunités de communications. Il convient néanmoins de pondérer la réalité de ces opportunités. Tout d'abord, cet équipement s'appelle le Stade de France et non le « Stade de la Seine-Saint-Denis $»^{10}$. Par conséquent, l'ancrage territorial de ce stade dans le département n'est pas immédiatement perceptible. L'opportunité offerte aux élus de s'approprier les valeurs positives associées aux événements qui s'y déroule et de communiquer par leur intermédiaire est donc finalement limitée. En outre, le Stade de France correspond en réalité à un « territoire dans le territoire ", c'est-à-dire à un espace qui, bien que situé dans le département, n'entretient avec lui que très peu de liens économiques ou sociaux ${ }^{11}$. D'une certaine manière, cet équipement devait être un lieu d'intégration. Il est parfois davantage perçu comme un lieu de ségrégation par la population locale qui ne dispose pas des ressources financières pour acheter des places permettant d'assister aux événements.

La gestion de cet équipement, assurée par un consortium composé d'intérêts privés et publics nationaux, souligne d'ailleurs assez bien la place finalement marginale occupée par les élus locaux de ce département. Pour ces raisons, et les entretiens l'ont confirmé, les acteurs publics locaux ne communiquent pas à travers les manifestations dites « récurrentes $»^{12}$ qui y sont organisées. L'opportunité en trompe-l'œil que constituent ces événements « ordinaires » organisés au Stade de France conduit les élus locaux à un rôle de « spectateur ». Cette situation est caractérisée par l'existence de supports potentiels de communication que les élus locaux ne veulent pas ou ne sont pas en mesure d'exploiter dans le cadre de leur stratégie. La présence dans le département de ce stade pourrait même jouer un rôle contre-productif pour les élus locaux en accroissant le sentiment de rejet ressenti par une partie de la population locale.

Ce constat peut d'ailleurs être observé dans d'autres territoires : à Paris où l'extension du Stade Roland-Garros, qui constitue l'une des vitrines de la ville, fait l'objet de vives oppositions de la part de la population locale ${ }^{13}$ mais aussi à Pékin ou la construction des équipements nécessaires à l'organisation des JO de 2008 a nécessité le déplacement voire la destruction de quartiers entiers d'habitation ou à Londres où la reconstruction du Stade de Wembley a nécessité de redessiner profondément la physionomie des quartiers alentours. Dans tous ces cas, la stratégie de communication des acteurs publics locaux fondée sur ces équipements structurants peut s'avérer positive du point de vue de la communication externe (sous réserve d'en mesurer précisément l'efficacité) mais négative pour une partie de la population locale subissant les externalités négatives de tels projets.

\footnotetext{
${ }^{10}$ En outre, si une collectivité publique devait être identifiée comme un partenaire structurellement associé au Stade de France, c'est davantage la région lle-de-France qui ressortirait. Le conseil régional est en effet partenaire officiel du Stade de France depuis avril 2005 pour un montant annuel d'environ 1,5 million d'euros. Dans tous les cas, la Seine-Saint-Denis n'apparaît pas comme un partenaire direct du Stade de France.

${ }^{11}$ Certains cadres du Stade de France vont même plus loin en considérant que le principal handicap au développement de l'activité économique du Stade de France (vente de prestations haut de gamme pour les événements, de packages) est qu'il soit situé en Seine-Saint-Denis. Certains clients refusent l'achat de ces prestations en raison de cette localisation.

${ }^{12}$ Les manifestations « récurrentes » ou « ordinaires » sont celles qui sont organisées chaque année, qui ne présentent pas un caractère exceptionnel de part leur rareté ou leur dimension internationale.

133000 personnes ont signé la pétition contre l'extension du projet d'extension du stade. Des recours ont également été déposés.
} 


\subsection{L'acteur public « opportuniste »}

Le second rôle stratégique est celui « d'opportuniste ». Il se caractérise par la volonté des acteurs publics locaux de mettre en œuvre une communication par le sport en réaction à une opportunité qui se présenterait sans que ces acteurs publics en soient à l'origine. Les entretiens réalisés montrent que la Coupe du Monde de football 1998 est un bon exemple pour la Seine-Saint-Denis. C'est la France, via le comité ayant porté sa candidature, qui a obtenu l'organisation de cette épreuve. L'obtention de cet événement s'est immédiatement transformée en opportunité de communication pour les élus locaux du fait des matches (les plus nombreux et les plus prestigieux) que le comité d'organisation a prévu au Stade de France, donc en Seine-Saint-Denis.

Toutefois, les entretiens réalisés ont confirmé la difficulté pour une collectivité publique de niveau intermédiaire, telle qu'un département, de communiquer à travers un événement aussi important qu'une Coupe du Monde de football. Le « rapport de force » symbolique, politique et économique entre l'événement et le territoire étant tellement en faveur du premier qu'il est difficile au second d'éviter un phénomène de cannibalisation. Ce phénomène consiste, pour l'événement, à saturer l'espace de communication et à annihiler toute velléité du territoire en la matière ${ }^{14}$. Même si ce point mériterait d'être confirmé par des recherches ultérieures, il semble que lorsqu'une manifestation est de trop grande ampleur, les enjeux politiques, juridiques, économiques, sociaux ou encore symboliques dépassent le cadre du territoire d'accueil. Dans ce cas, il est difficile pour ce dernier de mettre en œuvre une stratégie volontariste, cohérente et sans doute efficace d'exploitation de l'événement en termes de communication. Les élus n'ont dès lors plus d'autres choix que de saisir les rares opportunités leur permettant de véhiculer le message voulu et de tenter de montrer qu'ils sont quand même au cœur de l'événement. Au-delà de ce qu'ils sont susceptibles d'en faire, ce rôle « opportuniste » des élus correspond bien à l'idée d'essayer de saisir des opportunités sans en être les initiateurs.

\subsection{L'acteur public « accompagnateur »}

Le troisième rôle stratégique des acteurs publics locaux en matière de communication est celui «d'accompagnateur ». Il correspond également au troisième stade d'investissement des décideurs locaux en matière de communication territoriale par des événements sportifs. Ce rôle se caractérise par une volonté de la sphère publique locale d'exploiter un événement sportif (avant et/ou pendant). Les entretiens réalisés indiquent que, pour la Seine-Saint-Denis, les élus locaux ont adopté ce rôle « d'accompagnateur » durant la Coupe du Monde de Rugby 2007. Ils ont en effet adopté en amont de cette manifestation une série de dispositifs volontaristes (précisés dans les résultats) visant à tirer partie du lien entre cette Coupe du Monde et son territoire d'accueil en termes d'image et d'animation

\footnotetext{
${ }^{14}$ Durand la Coupe du Monde 1998, les observateurs ont souvent souligné que les nombreux médias internationaux venus à Paris « couvrir » la Coupe du Monde de football allaient rarement au-delà d'un reportage sur la Basilique Saint-Denis lorsqu'il s'agissait d'évoquer les à-côtés de l'événement. La présence de médias internationaux sur le territoire au cours d'une période longue ne constitue donc pas la garantie de pouvoir construire un message positif pour l'image du territoire.
} 
du territoire. Ce rôle des décideurs publics locaux va donc plus loin que la seule volonté de saisir une opportunité qui se présenterait. Dans le cas d'un rôle d'accompagnement, les acteurs publics saisissent cette opportunité et s'en servent comme l'élément de départ d'une démarche de communication.

\subsection{L'acteur public « moteur »}

Le quatrième rôle stratégique est celui de l'acteur public «moteur ». Il s'agit du degré d'investissement le plus important de la sphère publique locale. C'est dans cette configuration que se trouvent les collectivités territoriales créant par exemple leur propre manifestation à des fins, notamment, de communication territoriale. Plus généralement, ce rôle moteur définit l'action de l'élu local lorsqu'il fait preuve d'une forte pro-activité pour initier, développer ou exploiter une manifestation sportive, un club sportif professionnel, des activités de loisirs sportifs sur son territoire. S'agissant du département analysé et sur la base des entretiens réalisés, ce rôle «moteur » des élus locaux a été identifié lors de deux événements : les Championnats du monde d'athlétisme en 2003 et la Transat AG2R.

Pour le premier, le rôle moteur des élus locaux est perceptible dans le soutien au bon déroulement de la manifestation. Sans ce soutien financier du département, mais également des autres collectivités territoriales, elle n'aurait peut-être pas eu lieu en raison des difficultés à trouver les 57 millions d'euros nécessaires. Les acteurs publics ont donc été présents en amont de la manifestation pour en garantir le déroulement. Durant l'événement, différents dispositifs ont permis aux élus d'établir puis de consolider le lien entre le territoire et l'événement afin de tirer partie de retombées symboliques positives.

Ce rôle moteur est encore plus notable s'agissant du projet « Esprit 93 » de la Transat AG2R. Les élus locaux ont conçu et intégralement porté ce projet. Ils ont également conçu un dispositif de visibilité du département durant l'épreuve destinée à la population locale, aux professionnels de la communication ${ }^{15}$, aux amateurs de voile et plus généralement à tous ceux qui suivent l'actualité nationale ayant « reçu » le message du département à travers la couverture médiatique dont l'événement a fait l'objet. On peut également faire l'hypothèse, non-vérifiée au cours de cette recherche, que ce message est plus efficace dans la mesure où le département est, dans le cas de la Transat AG2R, un compétiteur à part entière alors que pour les autres événements évoqués, il n'était au mieux que partenaire.

Bien qu'il soit indispensable de tester cette analyse au travers d'autres cas, il semble exister quatre rôles stratégiques des élus locaux synthétisés dans le tableau 4. Ces quatre rôles peuvent être hiérarchisés en autant de stades correspondant au degré d'investissement des acteurs publics locaux; le rôle de «spectateur» correspondant au premier stade alors que le rôle « moteur » correspondant à l'investissement le plus important.

\footnotetext{
15 En 2006, la communication du département à travers la Transat AG2R a permis à la Seine-SaintDenis d'être nommée dans la catégorie des meilleures stratégies de communication menées par une collectivité territoriale lors des Trophées Sporsora qui regroupent chaque année les professionnels de la communication par le sport.
} 
Tableau 3 : Les quatre rôles stratégiques joués par les acteurs publics locaux dans le cadre d'une communication territoriale par le sport

\begin{tabular}{|c|l|l|}
\hline Stade & Rôle stratégique & \multicolumn{1}{c|}{ Définition } \\
\hline $\mathbf{1}$ & Spectateur & $\begin{array}{l}\text { Existence de supports potentiels de communication par le sport que } \\
\text { les élus locaux ne veulent pas ou ne sont pas en mesure d'exploiter } \\
\text { dans le cadre de leur stratégie }\end{array}$ \\
\hline $\mathbf{2}$ & Opportuniste & $\begin{array}{l}\text { Mise en ouvre, par les acteurs publics locaux, d'une communication } \\
\text { par le sport en réaction à une opportunité qui se présenterait sans } \\
\text { que ces acteurs publics en soient à l'origine }\end{array}$ \\
\hline $\mathbf{3}$ & Accompagnateur & $\begin{array}{l}\text { Exploitation, par les acteurs publics locaux, à moyen et long termes, } \\
\text { d'une opportunité de communication liée au sport }\end{array}$ \\
\hline $\mathbf{4}$ & Moteur & $\begin{array}{l}\text { Fort investissement des acteurs publics locaux pour créer, initier, } \\
\text { développer ou exploiter un support de communication lié au sport. }\end{array}$ \\
\hline
\end{tabular}

Au-delà de l'identification de ces quatre rôles stratégiques, les résultats de l'étude permettent de préciser le type de stratégie mise en œuvre par les élus de Seine-SaintDenis. Ils indiquent en particulier que cette communication par le sport repose a priori sur deux supports principaux très différents : d'une part, de grands événements sportifs organisés sur le territoire dont les droits sont détenus par des organisations sportives internationales et dont les élus locaux cherchent à être les partenaires dans le cadre de contrats «classiques » de sponsoring, d'autre part, un équipement sportif structurant à fort rayonnement, le Stade de France. Le premier support serait ainsi propice à des communications ponctuelles à plus forte visibilité liée au caractère exceptionnel des événements auxquels le territoire est associé.

Le second constituerait un «fil rouge» dans le message véhiculé par les acteurs locaux permettant d'éviter les interruptions dans la communication territoriale. Si on s'en tient à cette lecture, c'est une stratégie de diversification qui semble être menée par les acteurs publics locaux. Pour autant, il ressort des entretiens que le Stade de France ne constitue pas à proprement parler un levier réel de communication pour le territoire. En Seine-Saint-Denis, le stade peut susciter un sentiment de rejet plutôt qu'à un sentiment de fierté ou d'appartenance. Ailleurs, les nouveaux stades à Lille ou à Lyon rencontrent une forte opposition de la part d'une partie de population locale qui voit dans ces équipements une nuisance et une source potentielle de pression fiscale accrue. Il peut donc exister un décalage entre la volonté politique des élus locaux qui souhaitent faire de leur équipement un élément de prestige et de visibilité de leur territoire et la manière dont il est effectivement accueilli par la population. Pour ces raisons, le parti pris de cette recherche consistant à n'interroger que «l'émetteur » du message, c'est-à-dire les acteurs publics locaux, ne permet pas de juger de son efficacité. Un tel objectif ne pourrait être atteint qu'en interrogeant également « le récepteur ». Le Stade de France en tant qu'équipement sportif structurant ne constitue donc pas un support de communication mobilisé par les élus locaux. C'est donc davantage une stratégie de spécialisation autour de grands événements sportifs qui est développée par les acteurs locaux de Seine-Saint-Denis selon 
la typologie proposée par Hautbois, Desbordes (2008) qui identifient trois stratégies majeures : de diversification, de différenciation et de spécialisation.

De manière plus générale, si elle s'inscrit bien dans une problématique de marketing territorial telle qu'elle a été définie dans la littérature mentionnée au début de l'article, cette recherche n'aborde que l'aspect communication. En d'autres termes, l'amélioration de la « part de marché du territoire » évoquée par Houllier-Guibert (2009) ne peut être évaluée au terme de la communication conçue par la Seine-Saint-Denis durant 10 ans. Cette recherche confirme néanmoins la pertinence du sport, et en particulier de l'événementiel sportif, comme support de communication du territoire. En ce sens, la démarche des élus du département se présente clairement comme un processus volontariste confirmant l'importance ce que mentionne Lacour (2004) à propos du rôle pro-actif des acteurs locaux dans la création d'une dynamique territoriale.

Ce volontarisme s'illustre à travers le choix des supports utilisés pour communiquer (ici les événements sportifs) mais également celui de la cible visée. Ici, c'est nettement une stratégie de communication externe au territoire qui est conduite par les acteurs publics. Pour autant, la dimension générale de cette stratégie ne permet pas d'identifier plus finement, au travers des entretiens réalisés, un public-cible externe particulier. C'est tout à la fois les Français n'habitant pas en Seine-Saint-Denis que les médias (nationaux et internationaux) ou encore les acteurs économiques (notamment franciliens) qui semblent visés. Ce constat est sans doute l'indicateur d'un manque de maturité de cette stratégie de communication. Il inviterait également à étudier plus en détail la communication interne menée par les élus locaux. L'objectif serait d'identifier la cohérence entre communication interne et externe : redondances, contradictions éventuelles ou complémentarité...

Enfin, la difficulté à généraliser nos observations à d'autres territoires ne semble que partiellement préjudiciable. En effet, Peirce, Brent (2004) rappellent qu'il appartient à chaque territoire d'identifier ce qui lui est spécifique et ce qu'il pourra valoriser. Ce qui vaut donc pour l'un est donc difficilement transférable en l'état à l'autre. Même si la construction d'un modèle demeure la finalité, l'importance des singularités locales reste un préalable.

\section{Conclusion}

L'objectif de cette recherche était de caractériser le rôle des acteurs publics dans le développement d'une stratégie d'image d'un département pour laquelle on postule que le sport constitue un levier pertinent. Sans chercher à évaluer l'efficacité de cette stratégie qui devrait faire l'objet d'une recherche ultérieure, l'objectif était spécifiquement d'analyser la manière dont le sport était mobilisé dans un contexte territorial donné. Les résultats obtenus ont permis de définir la place (très limitée) du Stade de France et celle (beaucoup plus importante) des grands événements sportifs comme support de cette communication.

Cette utilisation du sport en vue de modifier l'image du département entre en résonance avec d'autres projets initiés par les acteurs publics du département. C'est le cas de l'Appel des 93, campagne mobilisant 93 personnalités de Seine-Saint-Denis visant à «faire changer le regard porté sur le département ». L'analyse des entretiens a permis de suggérer une typologie des rôles stratégiques des acteurs publics. Celle-ci reste largement à confirmer au travers de recherches futures portant sur d'autres cas et intégrant éventuellement d'autres échelles territoriales. Plusieurs nations semblent ainsi investir le 
sport au sens large entendu comme un vecteur de construction identitaire et de promotion de leur image : la Serbie (première participation à la Coupe du monde de football en 2010, victoire en Coupe Davis en 2010), la Pologne et l'Ukraine (Euro de football 2012), le Qatar (Coupe du monde de football 2022) ou encore la Turquie (GP de Formule 1, Championnats du monde d'escrime en 2009, de basket en 2010, candidate pour l'Euro de football 2016) pourraient constituer des cas féconds.

\section{Bibliographie}

ARKSEY H., KNIGHT P. (1999), Interviewing for social scientists An introductory resource with examples. London: Sage.

BAYEUX P., (2006), Les relations entre collectivités locales et clubs sportifs, PUS, Voiron.

BAYEUX P., (2009), Le maire et le sport, Berger-Levrault, Paris.

BERRETT T., SLACK T., (2001), A framework for the analysis of strategic approaches employed by non-profit sport organizations in seeking corporate sponsorship, Sport Management Review 4 (1), 21-46.

BLOOM P. N., (1997), Field of Marketing and Public Policy: Introduction and Overview, The Journal of Public Policy \& Marketing 16 (1), 126-128.

BOUINOT J., (2004), La ville intelligente, LGDJ, Paris.

BURGAY M., (2005), Innovation et repositionnement du secteur touristique en Province de Turin à l'horizon des JO d'hiver 2006, Colloque Sentedalps.

BURTON S., (1999), Marketing for public organizations: new ways, new methods», Public Management, vol.1, $\mathrm{n}^{\circ} 3$, p. 373-385.

BUURMAN H., (2001), Public policy marketing: marketing exchange in the public sector, European Journal of Marketing 35 (11/12), 1287-1300.

CHAPPELET J.L., (2006), Les politiques publiques d'accueil des grands événements sportifs. L'Harmathan, Paris.

CHAZAUD P., (1989), Le sport dans la commune, le département et la région, Berger-Levrault, Paris.

COHEN-BACRIE B., Territoires en promotion : développement local, marketing territorial et stratégie d'image, Weka, Paris.

EISENHARDT K.M., (1989), Building theories from case study research, Academy of Management Review 14(4), 532-550.

FERRAND A., CHANAVAT N., (2006). Le marketing territorial événementiel. In J.L. CHAPPELET (Ed.), Les politiques publiques d'accueil et d'organisation d'événements sportifs 72-85, L'Harmattan, Paris.

GARDE J.-L., (2005), La politique événementielle sportive en Rhône-Alpes : analyse prospective, Colloque Sentedalps.

GIRARD V.,(1999), Contribution à l'étude du marketing territorial : le cas des projets de ville et d'agglomérations françaises, thèse de doctorat en sciences de gestion, Université de Lyon 3.

GLASER B.G, STRAUSS A.L., (1967), The Discovery of Grounded Theory: Strategies for Qualitative Research, Aldine Publishing Company, Chicago.

GODRON J., (2003). Le territoire stratégique : nouveaux enjeux et clés de réussite du développement économique décentralisé, L'Harmattan, Paris.

HATEM F., (2007), Le marketing territorial : principes, méthodes et pratiques, Eyrolles, Paris.

HAUTBOIS C., DESBORDES M., (2008), Sport et marketing public, Economica, Paris.

HOULLIER-GUIBERT C-E., (2009), Les politiques de communication rennaises pour un positionnement européen, thèse de doctorat de l'Université 2. 
LACOUR C., (2004), 40 ans d'aménagement du territoire, La Documentation Française, Paris.

LEBRETON V., (2008), «L'événementiel sportif au service de la stratégie de communication de la ville de Rennes : une démarche de marketing territorial de projet », In C. Hautbois, M. Desbordes (Eds.), Sport et marketing public.143-158, Economica, Paris

MELA A., (2005), Les Jeux de Turin 2006 : héritage pour le développement territorial, Colloque Sentedalps.

NOISETTE J., VALLERUGO F., (1996), Le marketing des villes : un défi pour le développement stratégique, Éditions d'Organisation, Paris.

PEIRCE S., BRENT R.W., (2007), "National capital branding: A comparative case study of Canberra, Australia and Wellington, New Zealand”, Journal of Travel Tourism Marketing, 22 (3/4), 67-78.

REBILLARD S., (2007), « L'analyse identitaire, un enjeu pour les territoires », Cahiers Espaces 245, 20-23.

RICHELIEU A., (2008), «Combiner gestion de la marque et relations publiques dans une démarche stratégique : le cas du Rouge et Or de l'université Laval au Canada », In C. Hautbois M. Desbordes (Eds.), Sport et marketing public 238-250, Economica., Paris

SEPPEY F., (2005), Politique du tourisme et événements sportifs valaisans, Colloque Sentedalps.

SPERLING D., (1991). Le marketing des collectivités locales, Ecomédia, Paris.

SPERLING D., (1995). Le marketing des territoires et des collectivités locales, Milan-Midia, Toulouse.

TEXIER L., Valla J.-P., (1992), « Le marketing territorial et ses enjeux », Revue Française de Gestion, 87, 45-55.

TEXIER, L., (1999), «Une clarification de l'offre d'implantation en marketing territorial : produit de ville et offre de territoire », Revue d'Économie Régionale et Urbaine 5, 1021-1036.

TIEBOUT C., (1956), A pure theory of local expenditure , The Journal of Political Economy, 64 (5), 416-424.

YIN R.K., (2003). Case study research: design and methods ( $3^{\text {rd }}$ ed.). Sage Publications, Thousand Oaks, CA.

\section{Annexe A : dispositif méthodologique}

Le dispositif méthodologique de cette étude repose sur deux volets. Des entretiens ont été menés de manière exhaustive auprès de l'ensemble des acteurs publics impliqués durant la période de référence. Cette approche qualitative est au cœur du dispositif méthodologique employé. En parallèle, des données secondaires ont été recueillies que ce soit dans une logique exploratoire (collecte des informations pertinentes permettant la construction du guide d'entretien utilisé ensuite) ou confirmatoire (validation post-entretiens des actions engagées ou des mesures prises par les acteurs publics et évoquées par ces derniers au cours des entretiens).

Les personnes interviewées ont été retenues sur la base d'une double procédure (tableau 4) : i) examen et sélection des interlocuteurs pertinents sur la base de leur position dans l'organigramme du conseil général de Seine-Saint-Denis (Arksey, Knight, 1999); ii) principe de saturation du modèle c'est-à-dire lorsque l'information collectée au cours des entretiens devient redondante avec les entretiens précédents (Glaser, Strauss, 1967). Au cours de la phase de collecte de données, des entretiens supplémentaires ont été réalisés sur la base de deux critères principaux : 1) la qualité et la pertinence des informations obtenues; 2) le fait que ces informations pouvaient être confirmées.

Cette double procédure fournit une bonne validité des résultats et permet d'interviewer, pour chaque partie prenante, les sources les plus pertinentes par rapport à la problématique proposée. L'ensemble de ces entretiens ont été conduits en face-à-face. Le guide d'entretien (voir annexe B) a été développé et testé pour sa cohérence, sa logique et le fait qu'il couvre l'ensemble des éléments clés de la recherche. 
Trois principaux thèmes étaient ainsi abordés au cours des entretiens : i) présentation de l'acteur interrogé et ses responsabilités dans le fonctionnement de la collectivité; ii) analyse de la manière dont il a participé à la conception et à la mise en œuvre de la stratégie de communication; iii) sa perception de la manière dont cette stratégie de communication a permis de faire évoluer l'image du territoire. Dix entretiens ont ainsi été conduits entre septembre 2008 et octobre 2009 permettant d'interroger de manière exhaustive l'ensemble des acteurs publics locaux impliqués dans la stratégie de communication du département. Chaque entretien a duré entre 50 et 80 minutes et a ensuite été retranscrit puis codé. Ces entretiens, précisés dans le tableau ci-dessous, ont ensuite fait l'objet d'une analyse de contenu.

Tableau 4 : Liste des personnes interviewées

\begin{tabular}{|c|c|c|c|}
\hline Parties prenantes & Nom & Fonction occupée & Code \\
\hline $\begin{array}{l}\text { (1)- Acteurs publics en charge de la } \\
\text { communication du département }\end{array}$ & Maurice Chauvet & $\begin{array}{l}\text { Directeur de } \\
\text { la communication } \\
\text { de } 1998 \text { à } 2003\end{array}$ & PMC1 \\
\hline $\begin{array}{l}\text { (1)- Acteurs publics en charge de la } \\
\text { communication du département }\end{array}$ & Olivier Cessot & $\begin{array}{l}\text { Assistant du directeur } \\
\text { de la communication } \\
\text { (Patrick Maurieres) } \\
\text { depuis } 2003\end{array}$ & PMC2 \\
\hline $\begin{array}{l}\text { (2) - Acteurs publics en charge de la } \\
\text { politique sportive du département }\end{array}$ & Daniel Mongeau & $\begin{array}{l}\text { Vice-président en charge } \\
\text { du sport de } 1998 \text { à } 2001\end{array}$ & PMS1 \\
\hline $\begin{array}{l}\text { (2) - Acteurs publics en charge de la } \\
\text { politique sportive du département }\end{array}$ & Gilbert Roger & $\begin{array}{l}\text { Vice-président en charge } \\
\text { du sport de } 2001 \text { à } 2004\end{array}$ & PMS2 \\
\hline $\begin{array}{l}\text { (2) - Acteurs publics en charge de la } \\
\text { politique sportive du département }\end{array}$ & Emmanuel Constant & $\begin{array}{l}\text { Vice-président en charge } \\
\text { du sport de } 2004 \text { à } 2008\end{array}$ & PMS3 \\
\hline $\begin{array}{l}\text { (2) - Acteurs publics en charge de la } \\
\text { politique sportive du département }\end{array}$ & Azzedine Taïbi & $\begin{array}{l}\text { Vice-président en charge } \\
\text { du sport de } 1998 \text { à } 2004\end{array}$ & PMS4 \\
\hline $\begin{array}{l}\text { (3) - Agence de développement } \\
\text { économique de la Seine-Saint-Denis } \\
\text { ("Seine-Saint-Denis Avenir ») }\end{array}$ & Philippe Cressent & Président & ESEP1 \\
\hline $\begin{array}{l}\text { (3) - Agence de développement } \\
\text { économique de la Seine-Saint-Denis } \\
\text { (« Seine-Saint-Denis Avenir ») }\end{array}$ & Francis Dubrac & $\begin{array}{l}\text { Directeur et responsable } \\
\text { du projet Transat AG2R } \\
\text { pour la Seine-Saint-Denis }\end{array}$ & ESEP2 \\
\hline $\begin{array}{l}\text { (3) - Agence de développement } \\
\text { économique de la Seine-Saint-Denis } \\
\text { ( Seine-Saint-Denis Avenir ») }\end{array}$ & Jérôme Cordelier & Directeur depuis 2003 & ESEP3 \\
\hline $\begin{array}{l}\text { (4) - Direction des sports et des loisirs } \\
\text { du département }\end{array}$ & Richard Bouché & Directeur depuis 1998 & SLD \\
\hline
\end{tabular}


Des données secondaires ont également été étudiées. Plusieurs types de documents ont été collectés :

- sites internet des organisations concernées (CG 93, ville de Paris, région Ile-de-France, Comité d'organisation de la Coupe du Monde de Rugby, Noisy-Le-Grand, Comex 93

- 11 compte rendu de réunions de travail organisées durant la phase de préparation des événements sportifs accueillis en Seine-Saint-Denis durant la période étudiée

- 6 compte rendu d'assemblés générales du CG 93

- 5 discours d'élus ou des acteurs publics du département

- éléments de communication (affiches, publicités, flyers, etc.)

\section{Annexe B : guide d'entretien}

Thème 1. Présentation de l'acteur interviewé et de ses responsabilités

a. Quel est votre rôle au sein du département de Seine-Saint-Denis?

b. Quelles étaient vos responsabilités auparavant?

c. Au sein de la politique conduite par le département, quels sont vos objectifs et projets principaux au cours de mois à venir?

Thème 2. Analyse de la manière dont l'acteur a participé à la conception et à la mise en œuvre de la stratégie de communication du département

a. Selon, vous quelle image les groupes suivants ont de la Seine-Saint-Denis?

i. population locale

ii. population française qui n'habite pas en Seine-Saint-Denis

iii. grands médias nationaux

iv. leaders du secteur économique

a. Quelle image voulez-vous que la Seine-Saint-Denis ait?

b. Quelles sont selon vous les différentes solutions permettant de donner à la Seine-Saint-Denis cette image?

c. Quelle est la place du sport dans ce processus selon vous?

d. Jusqu'à présent, comment avez-vous utilisé le sport dans le cadre de cette communication territoriale?

e. Pour quelles raisons?

f. Votre stratégie était basée sur quels critères?

g. Comment les autres élus ou acteurs politiques locaux ont-ils pris part à cette stratégie?

e. Comment qualifieriez-vous votre rôle dans ce contexte?

Thème 3 . Sa perception de la manière dont cette stratégie de communication a permis de faire évoluer l'image du territoire

a. Selon vous, à quel stade de sa stratégie de communication le département est-il rendu?

b. Pensez-vous que cette stratégie est une stratégie gagnante ? Pourquoi? 
e. Selon vous, cette stratégie a-t-elle fait évoluer l'image que les parties prenantes suivantes ont du département?

i. population locale

ii. population française qui n'habite pas en Seine-Saint-Denis

iii. grands médias nationaux

iv. leaders du secteur économique

d. Comment faites-vous le lien entre cet éventuel changement de perception et votre stratégie de communication?

e. Quelle est la prochaine étape de cette stratégie?

\section{Annexe C : typologie des événements sportifs utilisés par la Seine-Saint-Denis dans leur communication territoriale}

Les manifestations sportives ayant constitué un relais de la communication de la Seine-Saint-Denis et ayant été mentionnés par les acteurs interviewés sont présentés dans le tableau ci-dessous.

Tableau 5 : Typologie des événements sportifs utilisés par la Seine-Saint-Denis dans leur communication territoriale

\begin{tabular}{|l|l|l|}
\hline $\begin{array}{l}\text { Lien avec l'événement } \\
\text { sportif }\end{array}$ & $\begin{array}{l}\text { Noms } \\
\text { de l'événement sportif }\end{array}$ & $\begin{array}{l}\text { Implication du département } \\
\text { de Seine-Saint-Denis }\end{array}$ \\
\hline $\begin{array}{l}\text { Territoire hôte d'un } \\
\text { événement sport }\end{array}$ & $\begin{array}{l}\text { Coupe du monde 1998 de } \\
\text { la FIFA } \\
\text { Championnats du monde } \\
\text { d'athlétisme 2003 } \\
\text { Coupe du monde de rugby } \\
2007\end{array}$ & $\begin{array}{l}\text { Territoire hôte des matches } \\
\text { organisés au Stade de France } \\
\text { Territoire hôte des matches } \\
\text { organisés au Stade de France } \\
\text { Territoire hôte des matches } \\
\text { organisés au Stade de France }\end{array}$ \\
\hline $\begin{array}{l}\text { Partenariat avec } \\
\text { un participant à } \\
\text { l'événement sportif }\end{array}$ & $\begin{array}{l}\text { Course à la voile Transat } \\
\text { AG2R en 2006 }\end{array}$ & $\begin{array}{l}\text { Le logo de la Seine-Saint- } \\
\text { Denis pouvait être vu sur la } \\
\text { tenue du skipper du bateau }\end{array}$ \\
\hline $\begin{array}{l}\text { Initiateur d'événements } \\
\text { sportifs internationaux }\end{array}$ & $\begin{array}{l}\text { Concours international } \\
\text { de sauts d'obstacles } \\
\text { en } 2008\end{array}$ & $\begin{array}{l}\text { Territoire hôte de l'événement } \\
\text { et dénomination d'une des } \\
\text { épreuves (« Grand Prix du } \\
\text { Conseil Général de la Seine- } \\
\text { Saint-Denis ») }\end{array}$ \\
\hline
\end{tabular}

